\title{
Analysis of the clinical diagnosis and treatment of uveitis
}

\author{
Lina Wang ${ }^{1,2 \#}$, Zhenfeng Guo ${ }^{1,3 \#}$, Yuezhong Zheng ${ }^{1,4}$, Qingyu Li ${ }^{1,4}$, Xiaoyong Yuan ${ }^{1,4}$, Xia Hua ${ }^{5}$ \\ ${ }^{1}$ Clinical College of Ophthalmology, Tianjin Medical University, Tianjin, China; ${ }^{2}$ Department of Ophthalmology, Xiaozhan Hospital, Tianjin, \\ China; ${ }^{3}$ Department of Ophthalmology, Beichen Hospital, Tianjin, China; ${ }^{4}$ Department of Ophthalmology, Tianjin Eye Hospital, Tianjin Key \\ Laboratory of Ophthalmology and Visual Science, Tianjin Eye Institute, Tianjin, China; ${ }^{5}$ Aier Eye Hospital, Tianjin, China \\ Contributions: (I) Conception and design: L Wang; (II) Administrative support: X Yuan; (III) Provision of study materials or patients: Y Zheng, L \\ Wang; (IV) Collection and assembly of data: L Wang, Z Guo; (V) Data analysis and interpretation: L Wang, Q Li; (VI) Manuscript writing: All \\ authors; (VII) Final approval of manuscript: All authors. \\ "These authors contributed equally to this work and should be considered as co-first authors. \\ Correspondence to: Xiaoyong Yuan, PhD. Clinical College of Ophthalmology of Tianjin Medical University; Tianjin Eye Hospital, Tianjin Key \\ Laboratory of Ophthalmology and Visual Science, Tianjin Eye Institute, Tianjin, China. Email: yuanxy_cn@hotmail.com.
}

Backgroundk Uveitis is a not uncommon potentially sight-threatening intraocular inflammatory disorder and a major cause of blindness worldwide. Early diagnosis and effective treatment are very important to reduce complications and protect vision.

Methods: In a retrospective series, we enrolled 263 (390 eyes) consecutive uveitis patients, comprising 126 males and 137 females. The clinical types of uveitis, treatment efficacy, complications and visual prognosis were evaluated by detailed clinical examination, laboratory tests and treatment observation.

Results: There were 101 cases of panuveitis (38.4\%), 85 cases of anterior uveitis (32.3\%), 75 cases of posterior uveitis $(28.5 \%)$, and 2 cases of intermediate uveitis $(8 \%)$. There was a statistically significant difference in (I) visual acuity between the acute and recovery periods ( $\mathrm{F}=13.12, \mathrm{P}<0.05)$; (II) visual acuity between first-time and recurrent patients $(\mathrm{F}=9.26, \mathrm{P}<0.05)$; (III) visual acuity in the affected and healthy eyes in the total, anterior and posterior uveitis groups $(\mathrm{P}<0.05)$; and (IV) the presence of ocular complications. There was also a statistically significant difference in the presence or absence of ocular complications between patients with initial disease and those with a recurrence of disease $(\mathrm{F}=59.51, \mathrm{P}<0.05)$.

Conclusions: In all its varying presentations, uveitis has a great impact on visual acuity. A careful clinical history should be taken and specific laboratory tests should be performed in order to improve the diagnosis and determine the etiology, and practitioners should provide appropriate treatment to avoid ocular complications, which can further damage visual acuity.

Keywords: Uveitis; diagnostics; treatments; outcomes; etiology

Submitted Oct 31, 2021. Accepted for publication Dec 16, 2021.

doi: 10.21037/apm-21-3549

View this article at: https://dx.doi.org/10.21037/apm-21-3549

\section{Introduction}

Uveitis is a not uncommon potentially sight-threatening intraocular inflammatory disorder that is a major cause of blindness worldwide $(1,2)$. The incidence is $20-50 / 100,000$ /year and the prevalence is $100-150 / 100,000$ (3). However, it varies in presentation and more contagious causes are found in less developed countries. Overall, uveitis causes $10-15 \%$ of global blindness, with $4-10 \%$ of patients blinded by uveitis in China, and is the third leading cause of preventable blindness in the world $(4,5)$. The etiology of uveitis is complex and varied, and the pathogenesis involves many aspects. Medical therapy is the cornerstone of the management of uveitis (6), because uncontrolled ocular inflammation can result in visually impactful ocular structural complications (7), and a significant risk of visual disability. Long-term treatment can have serious psychological, economic and quality-oflife consequences $(8,9)$; if timely diagnosis and treatment 
are not instituted, serious complications lead to irreversible damage of visual function. Clinicians should actively try to find the cause of uveitis to administer effective treatment, and for idiopathic uveitis, the best treatment plan to control the degree of inflammation and prevent complications and irreversible ocular structural damage and visual impairment should be selected according to the severity of the disease. We present the following article in accordance with the STROBE reporting checklist (available at https://dx.doi.org/10.21037/ apm-21-3549).

\section{Methods}

\section{Data collection}

Between January 2018 to June 2021, 263 consecutive patients seen at the specialty uveitis clinic of the Tianjin Eye Hospital with various types of uveitis were enrolled. Detailed ocular and medical histories were obtained and the patients underwent routine slit lamp microscopy and fundus examinations, and tonometry to measure their intraocular pressure (IOP). Furthermore, 168 patients underwent fluorescein fundus angiography, ocular color fluorescein fundus angiography, ocular color Doppler examination and optical coherent tomography. Complete blood count, erythrocyte sedimentation rate, anti-chain "O", rheumatoid factor, antinuclear antibody, human leukocyte antigen (HLA)-27, chest or sacroiliac joint X-ray, skin mycotoxin tuberculosis testing, and syphilis and the blood HIV antibody testing were performed as needed. All patients were followed up for more than 6 months. Visual acuity examinations were recorded using the $\log M A R$ scale to determine the best corrected visual acuity of the patients. All participants gave their written informed consent. The study was approved by the Institutional Ethics Committee of Tianjin Eye Hospital (No. 2021023). The study was conducted in accordance with the Declaration of Helsinki (as revised in 2013).

\section{Clinical classification}

The clinical classification and anatomically classified were based on the criteria of the International Uveitis Study Group, and the patients were classified as having anterior uveitis, intermediate uveitis, posterior uveitis or panuveitis according to the anatomic location. Based on the duration of the disease, acute uveitis was defined as duration of up to 3 months, chronic uveitis was defined as duration of more than 3 months, and recurrent uveitis was defined as the disease being stable for more than 3 months (10).

\section{Treatment}

For mild anterior uveitis, topical glucocorticoids $(0.5 \%$ tobramycin dexamethasone drops or $1 \%$ prednisolone acetate), compounded tropicamide to dilate the pupil and nonsteroidal anti-inflammatory (pralophine) drops were given, and a subconjunctival injection of dexamethasone sodium phosphate (3-4 mg) was given to patients with fibrin exudation in the anterior chamber. Patients with panuveitis and posterior uveitis were given oral prednisone tablets $(0.8-1.0 \mathrm{mg} / \mathrm{kg}$, decreasing every $7-14$ days until discontinuation depending on the condition). For patients with contraindications to oral prednisone tablets or recurrent disease, treatment with an immunosuppressant, cyclosporine A or azathioprine, and systemic treatment with adalimumab or infliximab was given for severe ocular inflammation. Oral calcium tablets were given to patients taking oral prednisone tablets to prevent calcium loss, and the patients taking oral prednisone were also given gastric protection medication to reduce gastrointestinal reactions. Oral potassium chloride tablets were given to prevent hypokalemia in patients who had immunosuppressive therapy. During the treatment period, routine blood tests, including liver and kidney function, were regularly evaluated.

\section{Statistical analysis}

Statistical analysis was performed using SPSS 11.5. Univariate analysis was performed using the independent sample $t$-test for the visual acuity of the affected eyes in the acute recovery period, and ANOVA was performed to determine the differences between the groups of patients with initial and recurrent uveitis, anterior uveitis, posterior uveitis and total uveitis. Variables were analyzed by a $2 \times 2$ correlation using the Pearson correlation test. Statistical significance was defined as $\mathrm{P}<0.05$.

\section{Results}

\section{Basic characteristics of the patients}

The 263 patients with various types of uveitis included a total of 390 eyes. The mean age of onset was $44.62 \pm 15.15$ years (range, 4-75 years). There were 126 males, with a mean age of onset of $42.84 \pm 15.82$ years, and 137 females, with a mean age of onset of $46.25 \pm 14.38$ years (male to female 
Table 1 Comparison of the visual acuity of affected eyes between two groups of patients in the acute phase recovery $(\bar{x} \pm \mathrm{S})$

\begin{tabular}{lccccc}
\hline Group & Eyes $(\mathrm{n})$ & Age $($ years $)$ & Mean visual acuity of affected eyes & $F$ value & $P$ value \\
\hline Acute phase & 159 & $44.84 \pm 15.21$ & $0.53 \pm 0.33$ & 13.122 \\
Recovery phase & 221 & $44.14 \pm 15.46$ & $0.73 \pm 0.28$ & \\
\hline
\end{tabular}

ratio $0.92: 1.00)$. There were 127 patients $(44.99 \%)$ with bilateral disease and 136 cases $(51.71 \%)$ of monocular disease. The mean visual acuity of the affected eye at the time of initial consultation was $0.63 \pm 0.31$ and that of the healthy eye was $0.77 \pm 0.29$. There were a total of 101 cases (38.4\%) of total uveitis, 85 cases (32.3\%) of anterior uveitis, 75 cases $(28.5 \%)$ of posterior uveitis, and 2 cases $(8 \%)$ of intermediate uveitis. There were 196 cases $(74.5 \%)$ of primary uveitis, 67 cases (25.5\%) of recurrent uveitis; 108 cases $(41.4 \%)$ of acute uveitis and 155 cases $(58.9 \%)$ of recovered uveitis. There were 132 cases $(50.2 \%)$ of topical treatment combined with oral glucocorticoids, 28 cases (10.6\%) of immunosuppressive treatment, and 15 cases (5.7\%) of treatment with biological agents.

\section{Etiological analysis}

According to the histories, clinical manifestations and laboratory findings, there were 105 patients (39.9\%) with idiopathic uveitis; 158 patients $(60.1 \%)$ with an identified etiology or an underlying systemic disease, including 39 cases $(14.8 \%)$ of Vogt-Koyanagi-Harada disease, 31 cases $(11.8 \%)$ of rheumatoid-associated uveitis, 20 cases of leukoaraiosis (7.6\%), 15 cases of HLA-B27 positive uveitis without systemic disease $(5.7 \%), 15$ cases of tuberculosis-associated uveitis (5.7\%), 12 cases of combined ankylosing spondylitis (4.6\%), 11 cases of juvenile arthritis combined with uveitis (4.2\%), 9 cases of viral uveitis (3.4\%), 5 cases of Fuchs syndrome (1.9\%), and 1 case of SAHPO syndrome (synovitis, acne, pustulosis, bone hypertrophy and osteomyelitis).

\section{Complications and visual impairment}

Various ocular complications occurred in 67 cases (25.5\%), including macular cystoid edema in 43 cases (16.3\%), incomplete posterior iris adhesions in 29 cases $(11.0 \%)$, secondary glaucoma in 28 cases (10.6\%), and concomitant cataracts in 25 cases (9.5\%). Adverse reactions associated with oral glucocorticoids occurred in $71(27.0 \%)$ patients, and included gastric discomfort in $29(11.0 \%)$ patients, facial, chest and back rash in 19 (7.2\%) patients, weight gain in $18(6.8 \%)$ patients, bone and joint discomfort in 18 (6.8\%) patients, mood effects and insomnia in $8(3.0 \%)$ patients, and fatigue in $5(1.9 \%)$ patients. Mild visual impairment (corrected visual acuity $<0.5$ ) was noted in 143 eyes $(36.7 \%)$, moderate and severe visual impairment $(<0.3)$ was seen in 84 eyes $(21.5 \%)$, and blinding $(<0.05)$ was seen in 12 eyes (3.1\%).

\section{Comparison of the visual acuity in acute, convalescent, primary and recurrent patients}

The corrected visual acuity of the patients was $0.53 \pm 0.33$ in the acute phase, and $0.73 \pm 0.28$ in the recovery phase, which was statistically significant in both groups $(\mathrm{F}=13.122$, $\mathrm{P}<0.05$ ) (Table 1). The corrected visual acuity at the last follow-up was $0.74 \pm 0.27$ in those patients with initial disease, and $0.62 \pm 0.32$ in those patients with a recurrence of uveitis, and the difference was statistically significant between the two groups $(\mathrm{F}=9.255, \mathrm{P}<0.05)$. There was a statistically significant difference in the presence of ocular complications between patients with an initial onset of uveitis and those with a recurrence of uveitis $(\mathrm{F}=59.51$, $\mathrm{P}<0.05)$; there were no statistically significant differences between the visual acuities in the healthy eyes, the IOP in the affected eyes and the IOP in the healthy eyes $(\mathrm{P}>0.05)$ (Table 2). There was a statistically significant difference between the corrected visual acuity $(0.58 \pm 0.24)$ in patients with idiopathic uveitis and the visual acuity $(0.70 \pm 0.32)$ in patients with uveitis associated with other diseases $(\mathrm{F}=6.825$, $\mathrm{P}=0.01)$.

\section{Comparison of the visual acuity and IOP between patients with different types of uveitis}

The differences in visual acuity between the affected and healthy eyes in the three groups of patients with total uveitis, anterior uveitis and posterior uveitis were statistically significant $(\mathrm{P}<0.05)$. There was no statistically significant difference in the IOPs between the affected and healthy eyes $(\mathrm{P}>0.05$, Table 3$)$. 
Table 2 Comparison of the visual acuity of affected eyes between first-time and recurrent patients $(\bar{x} \pm \mathrm{s})$

\begin{tabular}{lccccc}
\hline Group & $\begin{array}{c}\text { Visual acuity of } \\
\text { affected eye }\end{array}$ & $\begin{array}{c}\text { Visual acuity of } \\
\text { healthy eye }\end{array}$ & $\begin{array}{c}\text { Pressure in the } \\
\text { affected eye }\end{array}$ & $\begin{array}{c}\text { Pressure in the } \\
\text { healthy eye }\end{array}$ & $\begin{array}{c}\text { With or without ocular } \\
\text { complications }\end{array}$ \\
\hline Initial group $(\mathrm{n}=298)$ & $0.74 \pm 0.27$ & $0.70 \pm 0.29$ & $14.17 \pm 3.84$ & $14.73 \pm 3.67$ & $0.15 \pm 0.36$ \\
Recurrent group $(\mathrm{n}=92)$ & $0.62 \pm 0.32$ & $0.78 \pm 0.29$ & $14.97 \pm 4.14$ & $14.32 \pm 3.37$ & $0.32 \pm 0.47$ \\
F value & 9.255 & 0.019 & 1.065 & 1.807 & 0.182 \\
P value & 0.003 & 0.891 & 0.303 & 59.513 & 0.000 \\
\hline
\end{tabular}

Table 3 Comparison of the visual acuity and IOP between three groups of patients

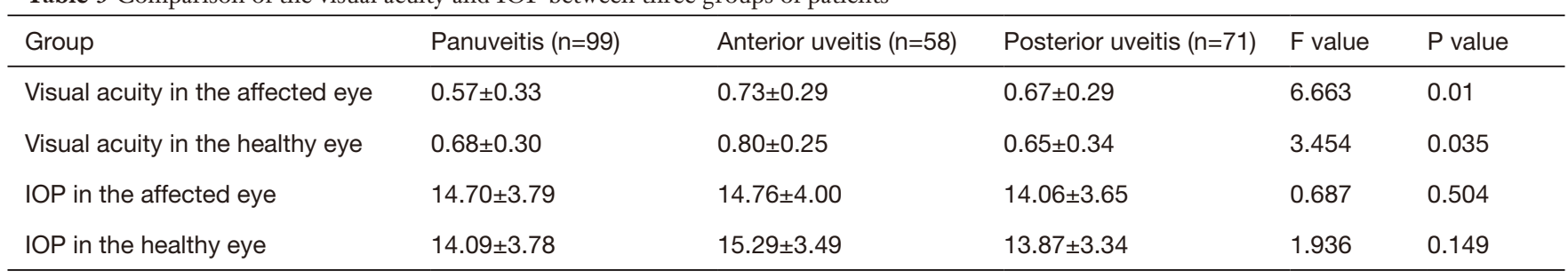

IOP, intraocular pressure.

\section{Results of correlation analysis}

There was a correlation between the visual acuity of the affected eye with the presence of vitreous inflammatory cells $(\mathrm{r}=0.174, \mathrm{P}=0.005)$.

\section{Discussion}

Uveitis is a group of diseases, with an estimated annual incidence of 38-715 per 100,000 people in developed countries (1). In Swiss, Hoogewoud et al. in the past 20 years (between 1 January 2000 and 31 December 2019) found that the incidence of pediatric uveitis is $11 \%(317 / 2,468)(11)$. The disease mainly invades the uveal tissue and causes an intraocular inflammatory response that can cause severe visual impairment. Uveitis accounts for $10-15 \%$ of the legally blind patients in the USA and up to $15 \%$ of the legally blind patients in other countries around the world (12-14). In this study, the incidence of mild visual impairment was $36.7 \%$, moderate and severe visual impairment reached $21.5 \%$, and the blinding rate was $3.1 \%$. The comparison of the visual acuities of the affected eyes and the healthy eyes among three groups (total uveitis, anterior uveitis and posterior uveitis) was statistically significant, and the visual acuities of the affected eyes and the healthy eyes in patients with anterior uveitis were better than those of patients with posterior and total uveitis. The visual acuity of patients with recovered uveitis was better than that of patients with acute uveitis, patients with primary uveitis were better than those with recurrent uveitis, and there was a correlation between the visual acuity of the affected eye and the presence of ocular complications, indicating the severity of the disease, the degree of inflammation, and those ocular complications most likely to damage the eye organs such as iris, lens, macula, optic nerve and intraocular pressure and affect visual acuity.

Diagnosing the etiology of uveitis is crucial in determining the treatment and prognosis, but no clear cause is found in approximately $28-45 \%$ of cases $(14,15)$. Statistically, idiopathic uveitis accounts for $20-40 \%$ of cases and infectious uveitis accounts for $30-50 \%$ of cases. However, in developed countries, infectious uveitis accounts for approximately $20 \%$, and the most common cause is herpesvirus infection (16). Sharma et al. (17) found that uveitis is associated with systemic diseases. Nelson et al. (18) reported that a specific etiological diagnosis was made in around $61 \%$ of patients, and in this study, the etiology was identified or combined with systemic diseases in $60.1 \%$ of the patients. It has been reported in the literature that rheumatic diseases predispose patients to the development of uveitis and damage to ocular tissues, and other diseases, such as Behcet's disease, syphilis, serosanguinous spondyloarthritis and psoriatic diseases, can also lead to inflammatory responses in the uvea (19). Grumet et al. (20) found that the most common systemic diseases associated with uveitis 
included spondyloarthritis and HLA-B27-associated uveitis, tuberculosis, and concurrent tuberculosis and herpesvirus infectious uveitis. These five types of uveitis accounted for $70 \%$ of all patients, followed by Behcet's disease and syphilis with incidences of $4.2 \%$ and $1.7 \%$, respectively. In a study by Gogia et al. (21), tuberculosis of the lung was found in $75 \%$ of the patients with tuberculous uveitis, anterior uveitis was found in $48.9 \%$ of the patients with tuberculous uveitis, posterior uveitis was found in $25.5 \%$ of the patients, total uveitis was found in $10.6 \%$ of the patients and intermediate uveitis was found in $10.6 \%$ of the patients. Multifocal chorioretinitis was the most common type of posterior uveitis. In this study, uveitis was present with systemic disease in 158 cases $(60.1 \%)$, including rheumatoidassociated uveitis in $11.8 \%$ of patients, leukoarthritis in $7.6 \%$ of patients, HLA-B27 positive and tuberculosis-associated uveitis without systemic disease in $5.7 \%$ each of patients, combined with ankylosing spondylitis in $4.6 \%$ of patients, juvenile arthritis combined with uveitis in $4.2 \%$, and $3.4 \%$ of viral uveitis.

These findings suggest that patients with uveitis should be actively tested for different etiologies, and that the combination of etiologic and symptomatic treatment results in a faster recovery and better outcomes. Other studies have shown that stress and lack of sleep may contribute to the recurrence of anterior uveitis, while exercise, smoking, and alcohol consumption are not associated with recurrence (22). Other researchers have found that air pollution leads to increased levels of inflammatory cytokines and exacerbates the autoimmune inflammatory response (23).

For the treatment of uveitis, topical and systemic administration of glucocorticoids can control the inflammatory response, especially in the early stages of inflammation when rapid control of the disease is essential, and because long-term usage of glucocorticoids can cause serious side effects, patients with recalcitrant uveitis often need to be treated with a combination of immunomodulatory agents (24). Guidelines for the treatment of uveitis include glucocorticoids as first-line therapy, immunomodulatory drugs such as cyclosporine A, methotrexate, azathioprine, and Mycophenolate Mofetil as second-line therapy, and biological agents [tumor necrosis factor-alpha antagonists (TNF- $\alpha$ ) or interferon-alpha] as third-line therapy $(24,25)$. Neti et al. (22) found that slowrelease glucocorticoid injections were rapidly effective in improving visual acuity, with $86 \%$ improvement in visual acuity, $27 \%$ improvement in pain and 26\% improvement in vitreous inflammatory clouding. Posarelli et al. (26) found that systemic steroids were used in $42.86 \%$ of the patients with uveitis, and the most common ocular complications were cataracts and an increased IOP, but there was no statistical correlation with systemic steroid application. Pavesio et al. (27) found that the use of $0.2 \mu \mathrm{g} / \mathrm{day}$ fluocinolone acetate implant to prevent the inflammation of posterior non-infectious uveitis reduced the recurrence and need for adjuvant medication, and improved vision, providing greater protection against ocular inflammation than a reactive approach using standard of care. It is more suitable for patients with uveitis recurrence $\geq 2$ times a year who need systemic corticosteroids or immunosuppressive therapy. Control rates of $52 \%$ for uveitis after 12 months of cyclosporine treatment, $60-76 \%$ for azathioprine and methotrexate, $69 \%$ for tacrolimus, and $79 \%$ for adalimumab have been previously reported; anti-TNF- $\alpha$ agents are particularly effective in patients with idiopathic uveitis without systemic inflammatory diseases (28). Suhler reported that for patients receiving subcutaneous adalimumab $40 \mathrm{mg}$ every other week, quiescence increased from $34 \%(122 / 364)$ at week 0 to $85 \%(153 / 180)$ at week 150 and corticosteroid use was reduced (29). In addition, anti-TNF- $\alpha$ agents can treat juvenile rheumatoid-associated uveitis, control the acute phase inflammatory response and disease severity, and have good efficacy for macular edema secondary to uveitis (30). Silvestri et al. found that adalimumab not only reduced macular edema in patients with uveitis but also controlled the active inflammatory response in systemic diseases (31). Puyade et al. performed autologous hematopoietic stem cell transplantation for Behçet's disease (BD) and reported an acceptable safety profile for a feasible and relatively effective procedure in severe and conventional treatment-resistant cases of $\mathrm{BD}$ that has the potential to stabilize BD in patients with life-threatening involvement (32). In our study, different treatment regimens were selected according to the severity of the patients' disease and systemic conditions, and included topical treatments combined with oral hormone therapy in 132 cases (50.2\%), supplemented with immunosuppressant cyclosporine $\mathrm{A}$ in 28 cases (10.6\%), and combined with adalimumab in 15 cases $(5.7 \%)$. We found a correlation between the visual acuity of the affected eye, the presence of ocular complications, and recovery. The visual acuity in idiopathic uveitis was statistically significant compared with the visual acuity in other types of diseaseassociated uveitis.

Therefore, the key aim of treatment is to control the degree of inflammation and prevent irreversible destruction 
of the ocular structures and subsequent visual damage. In conclusion, because the etiologies of uveitis are complex, the inflammatory response is variable, and untimely treatment can cause serious visual impairment, every ophthalmologist is responsible for improving treatment techniques, making early and correct judgments about the history and symptoms, giving patients the best treatment options, shortening the course of the disease to reduce pain, and restoring the visual function as soon as possible. At the same time, ophthalmologists, in conjunction with internists, should conduct relevant laboratory tests to identify the cause of the disease, and joint symptomatic treatment improves the outcomes of uveitis. In addition, health education guidance must be given to patients with systemic diseases susceptible to uveitis, and early treatment is given in case of ocular discomfort to avoid further damage to visual function due to disease recurrence.

\section{Acknowledgments}

We thank all the patients who participated in this study. Funding: This work was supported by the National Natural Science Foundation of China (81970772, 81670817, 81670816, 81870638).

\section{Footnote}

Reporting Checklist: The authors have completed the STROBE reporting checklist. Available at https://dx.doi. org/10.21037/apm-21-3549

Data Sharing Statement: Available at https://dx.doi. org/10.21037/apm-21-3549

Conflicts of Interest: All authors have completed the ICMJE uniform disclosure form (available at https://dx.doi. org/10.21037/apm-21-3549). The authors have no conflicts of interest to declare.

Ethical Statement: The authors are accountable for all aspects of the work in ensuring that questions related to the accuracy or integrity of any part of the work are appropriately investigated and resolved. All participants gave their written informed consent. The study was approved by the Institutional Ethics Committee of Tianjin Eye Hospital (No. 2021023). The study was conducted in accordance with the Declaration of Helsinki (as revised in 2013).
Open Access Statement: This is an Open Access article distributed in accordance with the Creative Commons Attribution-NonCommercial-NoDerivs 4.0 International License (CC BY-NC-ND 4.0), which permits the noncommercial replication and distribution of the article with the strict proviso that no changes or edits are made and the original work is properly cited (including links to both the formal publication through the relevant DOI and the license). See: https://creativecommons.org/licenses/by-nc-nd/4.0/.

\section{References}

1. Tsirouki T, Dastiridou A, Symeonidis C, et al. A Focus on the Epidemiology of Uveitis. Ocul Immunol Inflamm 2018;26:2-16.

2. de Smet MD, Taylor SR, Bodaghi B, et al. Understanding uveitis: the impact of research on visual outcomes. Prog Retin Eye Res 2011;30:452-70.

3. Miserocchi E, Modorati G, Mosconi P, et al. Quality of life in patients with uveitis on chronic systemic immunosuppressive treatment. Ocul Immunol Inflamm 2010;18:297-304.

4. Krishna U, Ajanaku D, Denniston AK, et al. Uveitis: a sight-threatening disease which can impact all systems. Postgrad Med J 2017;93:766-73.

5. Gui W, Dombrow M, Marcus I, et al. Quality of Life in Patients with Noninfectious Uveitis Treated with or without Systemic Anti-inflammatory Therapy. Ocul Immunol Inflamm 2015;23:135-43.

6. Dick AD, Rosenbaum JT, Al-Dhibi HA, et al. Guidance on Noncorticosteroid Systemic Immunomodulatory Therapy in Noninfectious Uveitis: Fundamentals Of Care for UveitiS (FOCUS) Initiative. Ophthalmology 2018;125:757-73.

7. Lee JJY, Duffy CM, Guzman J, et al. Prospective Determination of the Incidence and Risk Factors of New-Onset Uveitis in Juvenile Idiopathic Arthritis: The Research in Arthritis in Canadian Children Emphasizing Outcomes Cohort. Arthritis Care Res (Hoboken) 2019;71:1436-43.

8. Cassedy A, Altaye M, Andringa J, et al. Assessing the validity and reliability of the Effects of Youngsters' Eyesight on Quality of Life (EYE-Q) questionnaire among children with uveitis. Arthritis Care Res (Hoboken) 2020. [Epub ahead of print]. doi: 10.1002/acr.24491.

9. Río LEC, Cheja-Kalb R. Psychosocial and economic impact of uveitis in Mexico. Gac Med Mex 
2021;157:140-6.

10. Trusko B, Thorne J, Jabs D, et al. The Standardization of Uveitis Nomenclature (SUN) Project. Development of a clinical evidence base utilizing informatics tools and techniques. Methods Inf Med 2013;52:259-65, S1-6.

11. Hoogewoud F, Cohen J, Rossi D, et al. Epidemiology of Childhood Uveitis in a Tertiary Care Center: A 20-Year Study. Klin Monbl Augenheilkd 2021;238:469-73.

12. Suttorp-Schulten MS, Rothova A. The possible impact of uveitis in blindness: a literature survey. Br J Ophthalmol 1996;80:844-8.

13. Bodaghi B, Cassoux N, Wechsler B, et al. Chronic severe uveitis: etiology and visual outcome in 927 patients from a single center. Medicine (Baltimore) 2001;80:263-70.

14. de Parisot A, Kodjikian L, Errera MH, et al. Randomized Controlled Trial Evaluating a Standardized Strategy for Uveitis Etiologic Diagnosis (ULISSE). Am J Ophthalmol 2017;178:176-85.

15. Barisani-Asenbauer T, Maca SM, Mejdoubi L, et al. Uveitis- a rare disease often associated with systemic diseases and infections- a systematic review of 2619 patients. Orphanet J Rare Dis 2012;7:57.

16. Miserocchi E, Fogliato G, Modorati G, et al. Review on the worldwide epidemiology of uveitis. Eur J Ophthalmol 2013;23:705-17.

17. Sharma SM, Jackson D. Uveitis and spondyloarthropathies. Best Pract Res Clin Rheumatol 2017;31:846-62.

18. Nelson WW, Lima AF, Kranyak J, et al. Retrospective Medical Record Review to Describe Use of Repository Corticotropin Injection Among Patients with Uveitis in the United States. J Ocul Pharmacol Ther 2019;35:182-8.

19. Abaño JM, Galvante PR, Siopongco P, et al. Review of Epidemiology of Uveitis in Asia: Pattern of Uveitis in a Tertiary Hospital in the Philippines. Ocul Immunol Inflamm 2017;25:S75-80.

20. Grumet P, Kodjikian L, de Parisot A, et al. Contribution of diagnostic tests for the etiological assessment of uveitis, data from the ULISSE study (Uveitis: Clinical and medicoeconomic evaluation of a standardized strategy of the etiological diagnosis). Autoimmun Rev 2018;17:331-43.

21. Gogia V, Venkatesh P, Garg SP, et al. Patterns of uveitis in patients with proven systemic (pulmonary and extrapulmonary) tuberculosis. Int Ophthalmol 2019;39:1665-7.

22. Neti N, Pimsri A, Boonsopon S, et al. Triggering factors associated with a new episode of recurrent acute anterior uveitis. Sci Rep 2021;11:12156.
23. van Eeden SF, Tan WC, Suwa T, et al. Cytokines involved in the systemic inflammatory response induced by exposure to particulate matter air pollutants $(\mathrm{PM}(10))$. Am J Respir Crit Care Med 2001;164:826-30.

24. Valdes LM, Sobrin L. Uveitis Therapy: The Corticosteroid Options. Drugs 2020;80:765-73.

25. Schwartzman S, Schwartzman M. The Use of Biologic Therapies in Uveitis. Clin Rev Allergy Immunol 2015;49:307-16.

26. Posarelli C, Talarico R, Vella G, et al. Do systemic steroids increase the risk of ocular complication in uveitis patients? Focus on a Italian referral center. Clin Rheumatol 2019;38:2917-23.

27. Pavesio C, Heinz C. Non-infectious uveitis affecting the posterior segment treated with fluocinolone acetonide intravitreal implant: 3-year fellow eye analysis. Eye (Lond) 2021. [Epub ahead of print]. doi: 10.1038/s41433-02101608-9.

28. Suhler EB, Adán A, Brézin AP, et al. Safety and Efficacy of Adalimumab in Patients with Noninfectious Uveitis in an Ongoing Open-Label Study: VISUAL III. Ophthalmology 2018;125:1075-87.

29. Suhler EB, Jaffe GJ, Fortin E, et al. Long-Term Safety and Efficacy of Adalimumab in Patients with Noninfectious Intermediate Uveitis, Posterior Uveitis, or Panuveitis. Ophthalmology 2021;128:899-909.

30. Skurkovich B, Skurkovich S. Inhibition of IFN-gamma as a method of treatment of various autoimmune diseases, including skin diseases. Ernst Schering Res Found Workshop 2006;(56):1-27.

31. Silvestri E, Bitossi A, Bettiol A, et al. Adalimumab effectively controls both anterior and posterior noninfectious uveitis associated with systemic inflammatory diseases: focus on Behçet's syndrome. Inflammopharmacology 2020;28:711-8.

32. Puyade M, Patel A, Lim YJ, et al. Autologous Hematopoietic Stem Cell Transplantation for Behçet's Disease: A Retrospective Survey of Patients Treated in Europe, on Behalf of the Autoimmune Diseases Working Party of the European Society for Blood and Marrow Transplantation. Front Immunol 2021;12:638709.

(English Language Editor: K. Brown)

Cite this article as: Wang L, Guo Z, Zheng Y, Li Q, Yuan X, Hua X. Analysis of the clinical diagnosis and treatment of uveitis. Ann Palliat Med 2021;10(12):12782-12788. doi: 10.21037/apm-21-3549 